\title{
Multiple Agents RendezVous In a Ring in Spite of a Black Hole
}

\author{
Stefan Dobrev* $\quad$ Paola Flocchini ${ }^{\dagger} \quad$ Giuseppe Prencipe $^{\ddagger} \quad$ Nicola Santoro $^{\S}$
}

\begin{abstract}
The Rendezvous of anonymous mobile agents in a anonymous network is an intensively studied problem; it calls for $k$ anonymous, mobile agents to gather in the same site. We study this problem when in the network there is a black hole: a stationary process located at a node that destroys any incoming agent without leaving any trace. The presence of the black hole makes it clearly impossible for all agents to rendezvous. So, the research concern is to determine how many agents can gather and under what conditions.

In this paper we consider $k$ anonymous, asynchronous mobile agents in an anonymous ring of size $n$ with a black hole; the agents are aware of the existence, but not of the location of such a danger. We study the rendezvous problem in this setting and establish a complete characterization of the conditions under which the problem can be solved. In particular, we determine the maximum number of agents that can be guaranteed to gather in the same location depending on whether $k$ or $n$ is unknown (at least one must be known for any nontrivial rendezvous). These results are tight: in each case, rendezvous with one more agent is impossible.

All our possibility proofs are constructive: we provide mobile agents protocols that allow the agents to rendezvous or near-gather under the specified conditions. The analysis of the time costs of these protocols show that they are optimal.

Our rendezvous protocol for the case when $k$ is unknown is also a solution for the black hole location problem. Interestingly, its bounded time complexity is $\Theta(n)$; this is a significant improvement over the $O(n \log n)$ bounded time complexity of the existing protocols for the same case.
\end{abstract}

Keywords: Mobile Agents, RendezVous, Gathering, Black Hole, Harmful Host, Ring Network, Asynchronous, Anonymous, Distributed Computing.

\section{Introduction}

In networked systems that support autonomous mobile agents, a main concern is how to develop efficient agent-based system protocols; that is, to design protocols that will allow a team of rather "simple" agents to cooperatively perform complex system tasks. A main approach to reach this goal is to break a complex task down into more elementary operations. Example of these primitive operations are wake-up, traversal, gathering, election. The coordination of the agents necessary to perform these operations is not necessarily simple or easy to achieve. In fact, the computational problems related to these operations are definitely non trivial, and a

\footnotetext{
${ }^{*}$ University of Ottawa, email:sdobrev@site.uottawa.ca

${ }^{\dagger}$ University of Ottawa, email:flocchin@site.uottawa.ca

¥Università di Pisa, email:prencipe@di.unipi.it

${ }^{\S}$ Carleton University, email:sdobrev@site.uottawa.ca
} 
great deal of theoretical research is devoted to the study of conditions for the solvability of these problems and to the discovery of efficient algorithmic solutions; e.g., see [4, 5, 6, 15].

At an abstract level, these environments, which we shall call distributed mobile systems, can be described as a collection $\mathcal{E}$ of autonomous mobile entities located in a graph $G$. Depending on the context, the entities are sometimes called robots or agents; in the following, we use the latter. The agents have computing capabilities and bounded storage, execute the same protocol, and can move from node to neighboring node. They are asynchronous, in the sense that every action they perform takes a finite but otherwise unpredictable amount of time. Each node of the network, also called host, provides a storage area called whiteboard for incoming agents to communicate and compute, and its access is held in fair mutual exclusion. The research concern is on determining what tasks can be performed by such entities, under what conditions, and at what cost.

In this paper, we focus on a fundamental task in distributed mobile computing, rendezvous in the simplest symmetric topology: the ring network. We will consider its solution in presence of a severe security threat: a black hole, a network site where a harmful process destroys all incoming agents without leaving a trace.

Rendezvous. The rendezvous problem consists in having all the agents gather at the same node; upon arriving there, each agent terminally sets its variable to arrived; there is no a priori restriction on which node will become the rendezvous point.

This problem (sometimes called gathering, point-formation, or homing) is a fundamental one in distributed mobile computing both with agents in graphs and with robots in the plane.

In the case of agents in the graph, the rendezvous problem has been extensively investigated focusing on more limited settings (e.g., without whiteboards) with two agents; e.g., see [1, 2, 3, $6,7,13,19]$. Almost from the start it became obvious that the possibility (and difficulty) of a solution is related to the possibility (and difficulty) to find or create an asymmetry in anonymous and symmetric settings, like the one considered here, to break symmetry in the problem and thus ensure a rendezvous solutions researchers have used randomization (e.g., [2]), or different deterministic protocols for the two agents (e.g., [19]), or indistinguishable tokens [13]. The case of more than two agents has been investigated in [11, 14, 17],

with only [11] providing a fully deterministic solutions for anonymous ring networks.

Let us stress that all these investigations assume synchronous agents and this assumption is crucial for the correctness of their solutions.

In contrast, in our setting, both nodes and agents, besides being anonymous, are also fully asynchronous.

The only known results for this setting are about the relationship between sense of direction and possibility of rendezvous [6]; interestingly, the link between rendezvous and symmetrybreaking is even more clear: rendezvous is in fact equivalent to the election problem [6].

Black Hole Location. Among the severe security threats faced in systems supporting mobile agents, a particularly troublesome one is a harmful host; that is, the presence at a network site of harmful stationary processes. The problem posed by the presence of a harmful host has been intensively studied from a programming point of view (e.g., see [12, 16, 18]), and recently also from an algorithmic prospective [8,9]. Obviously, the first step in any solution to such a problem must be to identify, if possible, the harmful host; i.e., to determine and report its location. Depending on the nature of the danger, the task to identify the harmful host might be difficult, if not impossible, to perform. 


\begin{tabular}{|c|c|c|c|c|}
\hline & \multicolumn{2}{|c|}{$n$ unknown, $k$ known } & \multicolumn{2}{|c|}{$n$ known, $k$ unknown } \\
\hline \multirow{2}{*}{ ORIENTED } & $\forall k$ & $R V(k-1)$ & $\forall k$ & $R V(k-2)$ \\
& & & & \\
\hline \multirow{3}{*}{ UNORIENTED } & k odd & $R V(k-2)$ & $k$ odd or $n$ even & $R V(k-2)$ \\
& $k$ even & $R V\left(\frac{k-2}{2}\right)$ & $k$ even and $n$ odd & $R V\left(\frac{k-2}{2}\right)$ \\
& $\forall k$ & $G(k-2,1)$ & $\forall k$ & $G(k-2,1)$ \\
\hline
\end{tabular}

Figure 1: Summary of possibility results.

A particularly harmful host is a black hole: a host that disposes of visiting agents upon their arrival, leaving no observable trace of such a destruction. The task is to develop a mobile agents protocol to determine and report the location of the black hole; the task is completed if, within finite time, at least one agent survives and knows the location of the black hole. The research concern is to determine under what conditions and at what cost mobile agents can successfully accomplish this task, called the black hole location problem. Note that this type of highly harmful host is not rare; for example, the undetectable crash failure of a site in a asynchronous network transforms that site into a black hole.

The black hole location problem has been investigated focusing on identifying conditions for its solvability and determining the smallest number of agents needed for its solution $[8,9,10]$. In particular, a complete characterization has been provided for ring networks [8].

Our Contributions. In this paper we consider the rendezvous problem in a more difficult setting: $k$ asynchronous anonymous agents dispersed in a totally symmetric ring network of $n$ anonymous sites, one of which is a black hole.

Clearly it is impossible for all agents to gather since an adversary (i.e., a bad scheduler) can immediately direct some agents towards the black hole. So, the research concern is to determine how many agents can gather. We study this problem and establish a complete characterization of the conditions under which the problem can be solved. The possibility results are summarized in the table shown in Figure 1; these results are tight: in each case, rendezvous with one more agent is impossible. It is interesting to observe that at least one of $k$ and $n$ must be known to the agents; however, knowledge of both is not necessary.

Some of these results are unexpected. For example, in an oriented ring all but one agents can indeed rendezvous even if the ring size $n$ is not known, a condition that makes black hole location impossible.

In an unoriented ring, at most $k-2$ agents can rendezvous; surprisingly, if they can not, there is no guarantee that more that $(k-2) / 2$ will. It is however always possible to bring all $k-2$ within distance 1 from each other.

All our possibility proofs are constructive: we provide mobile agents protocols that allow the agents to rendezvous or near-gather under the specified conditions.

Our rendezvous protocol, for the case when $k$ is unknown, is also a solution for the black hole location problem. Interestingly, its bounded time complexity is $O(n)$; this is a significant improvement over the $O(n \log n)$ bounded time complexity of the existing protocols for the same case $[8]$. 


\section{Definitions, Basic Properties and Techniques}

\subsection{The Framework}

The network environment is a ring $\mathcal{R}$ of $n$ anonymous (i.e., identical) nodes. Each node has two ports, labelled left and right; if this labelling is globally consistent, the ring will be said to be oriented, unoriented otherwise. Each node has a bounded amount of storage, called whiteboard.

In this network there is a set $a_{1}, \ldots, a_{k}$ of $k$ anonymous (i.e., identical) mobile agents. The agents can move from node to neighboring node in $\mathcal{R}$, have computing capabilities and bounded storage, obey the same set of behavioral rules (the "protocol"), and all their actions (e.g., computation, movement, etc) take a finite but otherwise unpredictable amount of time (i.e., they are asynchronous). Agents communicate by reading from and writing on the whiteboards; access to a whiteboard is done in mutual exclusion. The agents execute a protocol (the same for all agents) that specifies the computational and navigational steps. Initially, each agent is placed at a distinct node, called its homebase, and has a predefined state variable set to available. Let us denote by $x_{i}$ the homebase of agent $a_{i}$. Each homebase is initially marked by the corresponding agent.

The agents are aware of the fact that in the network there is a black hole $(\mathrm{BH})$; its location is however unknown. In this environment, we are going to consider the Rendezvous problem and the Near-Gathering problem defined below.

The Rendezvous problem $R V(p)$ consists in having at least $p \leq k$ agents gathering in the same site. There is no a priori restriction on which node will become the rendezvous point. Upon recognizing the gathering point, an agent terminally sets its variable to arrived. We consider a solution algorithm terminated when at least $p$ agents become arrived (explicit termination).

The Near-Gathering problem $G(p, d)$ consists in having at least $p$ agents within distance $d$ from each other. As for the Rendezvous problem we consider the algorithm terminated when at least $p$ agents know that they are within distance $d$ from each other and change their state to a terminal state. Clearly, $G(p, 0)=R V(p)$.

The efficiency of a solution protocol is obviously first and foremost measured in the size of the solution, i.e. the number of agents that the algorithm will make rendezvous at the same location. A secondary but important cost measure is the amount of time elapsed from the beginning to the termination of the algorithm. Since the agents are asynchronous, "real" time cannot be measured. We will use the traditional measure of bounded time, where it is assumed that the traversal of a link takes at most one time unit.

[STEFAN:] change here, might need to be refined. We can use also the 'latest agent to wake up', but the result is weaker and needs more work in adjusting the proofs and statements of lemmas and theorems. Usually, the time is measured from the moment the first agent wakes-up. However, in our setting the first agent to wake-up might immediately enter the black hole, and indeterminate amount of time may pass before other agents wake-up. In order to avoid this problem and keep the algorithms and analysis simple, we measure the time complexity from the moment the latest extremal agent wakes-up, where the extremal agents are defined as follows:

- In the oriented case, there is single extremal agent - the left-most one, measured from the $\mathrm{BH}$.

- In unoriented case, there are two extremal agents - the leftmost agent within each group of agents with the same understanding of left and right (note that 'leftmost' is evaluated using the orientation of the group). 


\subsection{Cautious Walk}

In the following we describe a basic tool, first introduced in [8], that we will use in all our protocols to minimize the number of agents that disappear in the back hole.

In our algorithms, the ports (corresponding to the incident links) of a node can be classified as (a) unexplored - if no agent has moved across this port, (b) safe - if an agent arrived via this port or (c) active - if an agent departed via this port, but no agent has arrived via it. Clearly, both unexplored and active links are dangerous in that they might lead to the black hole; the difference is that active links are being traversed, so there is in general no need for another agent to go through that link until the link is declared safe.

The technique we use, called cautious walk, is defined by the following two rules:

Rule 1. when an agent moves from node $u$ to $v$ via an unexplored port (turning it into active), it immediately returns to $u$ (making the port safe), and then goes back;

Rule 2. no agent leaves via an active port.

In the following, agents will either move only on safe links or move using cautious walk.

\subsection{Basic Results and Lower Bounds}

There are some basic obvious facts:

Theorem 2.1. In an anonymous ring with a black hole

1. $R V(k)$ is unsolvable;

2. If the ring is unoriented, then $R V(k-1)$ is unsolvable.

Proof.

1. Since the location of the back hole is unknown to the agents, the first agent that move could immediately disappear in the black hole and will never be able to gather.

2. Since there is no directional agreement, two agents could disappear in the black hole from both sides at their first move and will not be able to gather.

Rendez-vous problem $R V(p)$ is said to be non-trivial if $p$ is a non-constant function of $k$. Less obvious are the following facts.

Theorem 2.2. If $k$ is unknown, non-trivial rendez-vous requires locating the black hole.

Proof. If the black hole is not located, then there can be unexplored nodes (i.e., nodes whose ports are all unexplored) that can contain agents not participating to the gathering.

Furthermore,

Theorem 2.3. If $k$ is unknown, then $R V(k-1)$ cannot be solved.

Proof. Partition the agents into two equal size groups $A$ and $B$. Denote by $R_{A}$ and $R_{B}$ the regions of the ring explored by the agents $A$ and $B$ respectively. Since $k$ is unknown, the region $R_{A}$ must increase; w.l.o.g., let it first increase to the right (let $x$ be the first node visited in this exploration). Because of symmetry, also $B$ must first increase to the right (let $r(B)$ be the first node visited in this exploration).

If the next expansion of $A$ is again to the right (towards node $r(x)$ ), the adversary slows down this expansion to a halt. Within finite time, some agent in $A$ must be expanding to the left (towards node $l(A)$ ); the reason is that in a scenario where the agents in $B$ did not exist and the black hole were indeed in $r(x)$, the agents in $A$ would not be able to detect the black 
hole without proceeding in the opposite direction, contradicting Theorem 2.2. In fact, since the regions $R_{A}$ and $R_{B}$ are disjoint, for the agents in $A$ such a scenario is indistinguishable from the one where the two groups exist.

Consider now the setting of $A$ and $B$ as shown in Figure ??.

This means that at least one agent in $A$, moving towards $l(A)$ will enter the black hole. Consider now the execution of $B$; by symmetry the agents of $B$ will behave in the same way as the agents in $A$, hence, an agent in $B$ going right to $r(B)$ will also enter the black hole.

In view of the fact that knowledge of $n$ is necessary for locating a black hole

Theorem 2.4. [8] If $n$ is not known, black hole location is unsolvable.

it follows that

Theorem 2.5. Either $k$ or $n$ must be known for non-trivial rendez-vous.

Finally, let us state a general lower bound on the number of moves.

Theorem 2.6. Let $n$ be known, and the ring be unoriented. The $R V$ problem requires at least $\left\lfloor\frac{k}{2}\right\rfloor\left(n-\frac{k}{2}-2\right)$ moves.

Proof. Consider the situation when the agents are divided in two groups $a_{1}, \ldots a_{\left\lfloor\frac{k}{2}\right\rfloor}$ and $a_{-1}, \ldots a_{\left\lceil\frac{k}{2}\right\rceil}$ adjacent to the black hole. Let $x$ be an arbitrary RV point inside the segment between the two groups at distance $d$ from $a_{1}$. Regardless of the algorithm, $k-2$ agents have to move to the $R V$ point for a total of at least $\sum_{2}^{\left\lfloor\frac{k}{2}\right\rfloor}(|d-i|+|n-d-1-i|)$ moves. Solving, we obtain that the number of moves is at least $\left\lfloor\frac{k}{2}\right\rfloor\left(n-\left\lfloor\frac{k}{2}\right\rfloor-2\right)$.

\section{Characterization and Tight Bounds}

\subsection{RendezVous when $n$ is unknown}

An immediate consequence of the fact that $n$ is unknown is that, by Theorem $2.5, k$ must be known for non-trivial rendezvous to occur. Hence, in the rest of this section we assume that $k$ is known. Another consequence of $n$ being unknown is that, by Theorem 2.4, we can not locate the black hole!

Let us now examine under what conditions the problem can be solved and how.

\subsubsection{In Oriented Rings}

Theorem 3.1. $R V(k-1)$ can be always solved, and this can be achieved in time at most $3(n-2)$.

To prove this theorem, consider the following protocol GoRight; agents are in two states: explorer and follower.

\section{Protocol GoRight:}

1. Initially, everybody is an explorer.

2. An explorer moves right using cautious walk. If it enters a node visited by another agent, it becomes a follower.

3. A follower moves right, traversing only safe links. 
4. If there are $k-1$ followers in one node, the agents there terminate the execution of the protocol.

Lemma 3.2. Protocol GoRight solves $R V(k-1)$.

Proof. The rightmost agent before the black hole will remain explorer and eventually enter the black hole. All other agents eventually become followers. Since no follower will go past the last safe node explored by the sole remaining explorer, eventually all followers gather at one node.

Note that the rendezvous site is not necessarily next to the black hole.

Lemma 3.3. Protocol GoRight terminates in time at most $3(n-2)$ since the start of the leftmost agent.

Proof. Using cautious walk, the leftmost agent needs at most 3 time steps to traverse a link. Therefore, it will take it (and all other agents) at most $3(n-2)$ time steps to reach the rightmost safe node and rendezvous there.

Lemma 3.4. Protocol GoRight uses at most $n k-k^{2} / 2+2 n+o(n k)$ moves.

Proof. All moves can be divided into cautious walk and right progress. There are at most 2 cautious walk moves per edge, by all agents combined. An agent $i$ will make at most $s_{i}-1$ right progress moves, where $s_{i}$ is the distance of the $i$ 's starting location from the black hole, measured leftwards (the agent entering the black hole will make one more move). Since in each node at most one agent starts, all $s_{i}$ 's are different. Summing together yields the lemma.

Note: There are situations in which the $3(n-2)$ time bound is indeed achieved: Consider a scenario where there are agents in the two sites neighboring the black hole; the leftmost agent wakes up first and all other agents join the execution only when an agent arrives to their node. Clearly, the left most agent must wake-up all other agents, and every edge must be traversed using cautious walk.

Theorem 3.1 now immediately follows from Lemmas 3.2 and 3.3.

\subsubsection{Unoriented Rings}

Since the ring is not oriented, by Theorem 2.1, $R V(k-1)$ can not be solved as two agents can immediately disappear in the black hole. Hence, the best we can hope for is $R V(k-2)$. The result is rather surprising. In fact, either $k-2$ can gather or no more that $(k-2) / 2$ can, with nothing in between.

\section{Theorem 3.5.}

1. If $k$ is odd, $R V(k-2)$ can always be solved

2. If $k$ is even, $R V(p)$ can not be solved for $p>(k-2) / 2$; however, $R V((k-2) / 2)$ can always be solved.

3. $G(k-2,1)$ can always be solved.

To prove this theorem, we will logically partition the entities in two sets, "clockwise" (or blue) and "counterclockwise" (or red), where all entities in the same set have a common view of "right". Notice that each agent, although anonymous, can easily detect whether a message on a whiteboard has been written by an agent in the same set or not (e.g, each message contains also an indication of which of the two local ports the writer considers to be "right"). 
Consider first the case when $k$ is odd (recall $k$ is known).

We have the following protocol GR-ODD:

1. The agents of each set first of all execute the rendezvous algorithm GoRIGHT for oriented rings, independently of and ignoring the agents of the other set, terminating as soon as $(k-1) / 2$ follower agents of the same set gather in the same node.

(Notice: this will eventually happen, and only to one set, as there is only one set with at least $(k+1) / 2$ agents, and eventually only one of those agents will remain explorer).

Without loss of generality, let this happens to the red agents.

2. The node where the $(k-1) / 2$ red followers have gathered becomes the collection point, and one of the followers is selected as left-collector.

3. Every follower or blue explorer arriving at the collection point joins the group.

4. The left-collector $x$ travels (using cautious walk when necessary) left and tells every follower and red explorer it encounters to go to the collection point; it does so until it reaches the black hole or the last safe node explored by a blue explorer. In the latter case, the leftcollector leaves a message for the blue explorer $y$ informing it of the meeting point, and instructing it to become left-collector; it then returns to the collection point. If/when the explorer $y$ returns to that node, it finds the message, becomes left-collector and acts accordingly.

5. A red explorer returning to the collection point during its cautious walk (notice: there is only one) becomes now a right-collector.

6. The rules for the right-collector are exactly those for the left-collector, where "left" is replaced by "right", and viceversa.

Since $k$ is odd, we get

Lemma 3.6. There is only one collection point.

By construction of algorithm GR-ODD we have

Lemma 3.7. Every edge non-incident to the black hole will be traversed by a collector.

Because of cautious walk, at most 2 agents will enter the black hole; this fact, combined with Lemma 3.7, yields the following:

Lemma 3.8. $k-2$ agents will gather in the collection point.

Hence, by Lemmas 3.6 and 3.8, part (1) of Theorem 3.5 holds. Before proceeding with the proof of the other parts of Theorem 3.5, let us examine the time costs of Protocol GR-ODD.

Theorem 3.9. Protocol GR-ODd terminates in time at most $5(n-2)$.

Proof. Let $t_{1}$ be the time when the collection point was formed and let $s$ be the number of edges explored at time $t_{1}$. Since traversing one edge using cautious walk takes at most 3 time steps, we get $t_{1} \leq 3 s$. Let $t_{2}$ be the time by which every edge non-incident to the black hole has been traversed by a collector. We get $t_{2} \leq t_{1}+3(n-2-s)+s$, since traversing each of the $s$ already explored edges takes at most one time step. Finally, let $t_{3}$ be the time when all agents informed by the collectors arrive to the collection point. Clearly, $t_{3} \leq t_{2}+n-2 \leq 3(n-2)+s+n-2$. Since $s \leq n-2$, we get $t_{3} \leq 5(n-2)$. 
Theorem 3.10. Protocol GR-ODD uses at most $3 n k / 2-k^{2} / 4+O(n)+o(n k)$ moves.

Proof. By Lemma 3.4 the $(k-1) / 2$ followers that triggered the creation of the collection point have spent $n k / 2-k^{2} / 8+o(n k)$ moves. The collectors spend at most $2 n$ moves. The remaining $(k-1) / 2$ agents might have collected at the opposite end (spending $n k / 2-k^{2} / 8+o(n k)$ moves). Adding the $(n-2)(k-1) / 2$ moves sufficient to bring them to the collection point yields the theorem.

Consider now the case when $k$ is even (recall $k$ is known). To prove part (2) of Theorem 3.5 we first observe that $R V((k-2) / 2)$ can always be solved by trivially having each set execute the rendezvous algorithm GoRIGHT for oriented rings, and terminating it when at least $k / 2-1$ follower agents of the same set gather in the same node. To complete the proof, we need to show that, when $k$ is even, rendezvous of a greater number of agents can not be guaranteed.

Lemma 3.11. If $k$ is even then $R V(p)$ can not be solved for $p>(k-2) / 2$.

Proof. Assume first that $n$ is odd. Consider the symmetry axis passing through the $\mathrm{BH}$ and dividing the ring into two sides of equal size; denote by $\bar{v}$ the symmetric image of node $v$. Consider now a symmetric but "complemented" distribution of the agents on the two sides of the ring: if there is a blue agent in node $v$ then there is a red agent in node $\bar{v}$ (see Figure 2.a)); thus, to each agent $a$ with homebase $v$ corresponds an agent $\bar{a}$, of opposite color, with homebase $\bar{v}$. In this distribution, let both neighbors of the $\mathrm{BH}$ be occupied by agents. Consider now a synchronous execution (i.e., every move of an agent takes exactly one time step), where all agents start at the same time, and where the first move of the agents neighboring the $\mathrm{BH}$ be directed towards the $\mathrm{BH}$. Observe now that, to any move in a given direction by an agent $a$ it corresponds a move in the opposite direction by agent $\bar{a}$. In particular, whenever an agent $a$ crosses the symmetry axis, agent $\bar{a}$ will cross the axis in the opposite direction. Since after the first step there are $(k-2) / 2$ agents on each side (two agents went into the $\mathrm{BH}$ ), the number of agents on each side will always be $(k-2) / 2$.

If $n$ is even, choose the symmetry axis to be the line, dividing the ring into two sides of equal size, passing through the link between the $\mathrm{BH}$ and its clockwise neighbor $v$ (see Figure 2.b)). Force the link incident to $v$ to be very slow. Since the algorithm does not know $n$, it cannot distinguish this situation from the case of $n$ odd, and the same arguments as for $n$ odd would apply.

We now show that, although we cannot guarantee that more than half of the surviving agents rendezvous, we can however guarantee that all the surviving agents gather within distance 1 from each other. To prove this, we use the following protocol GR-EvEn.

First, each set of agents executes the rendezvous algorithm GoRIGHT for oriented rings, independently of and ignoring the agents of the other set, and terminate it when (at least) $k / 2-1$ follower agents of the same set gather in the same node. Notice that it is possible that two (but no more than two) such gathering points will be formed; further notice that they could be both made of agents of the same color!

Let us concentrate on one of them and assume, without loss of generalization, that it is formed of red agents. By definition, associated with it, there is a red explorer that will become a right-collector once it realizes the collection point has been formed; among the followers gathered there, a left-collector has also been selected. Both collectors behave as in GR-ODD except that, now, each of them could encounter a collector from the other group (if it exists). Therefore, we need to add the following rules: 


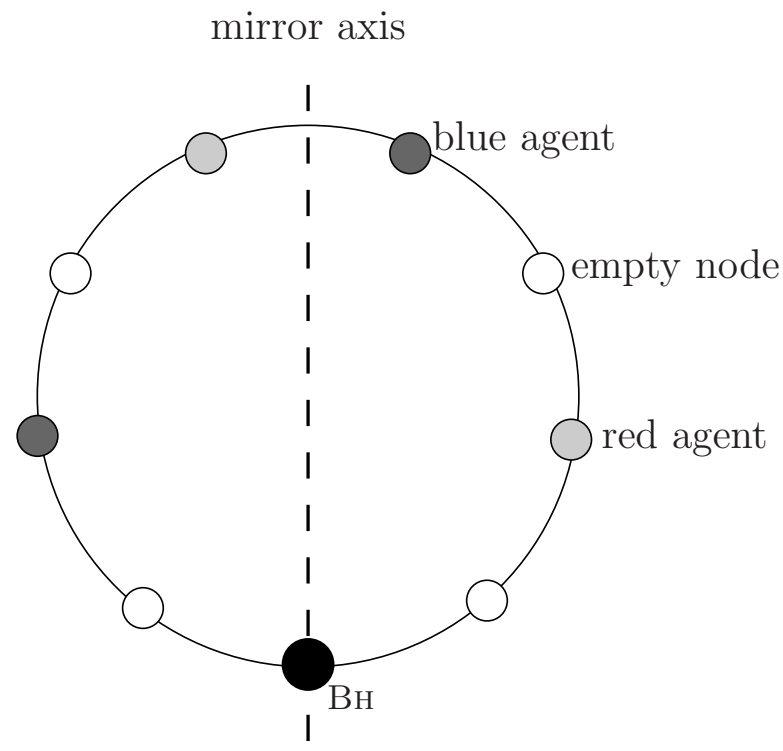

(a)

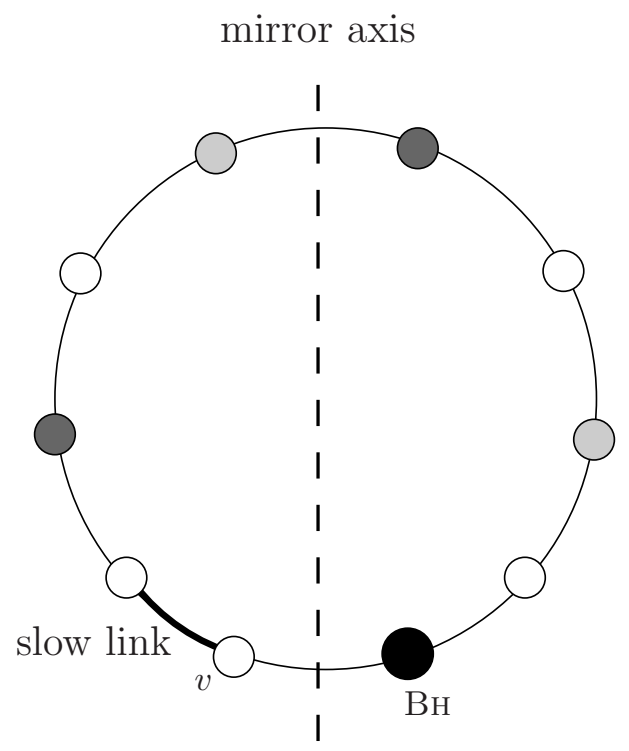

(b)

Figure 2: Mirror symmetric distribution for (a) $n$ odd, and (b) $n$ even.

1. a collector keeps the distance from its collection point. When passing the role of collector to an explorer, it passes also the distance information.

2. when a collector meets another collector (notice: they must be from different groups; further notice, they might actually "jump" over each other):

(a) if they are of the same color, then they agree on a unique site (e.g., the rightmost of the two ones) as the final common collection point;

(b) if they are of different colors, if the distance between the collection points is odd, they agree on the middle node as the final common collection point; otherwise, each chooses the closest site incident on the middle edge as the final collection point of its group.

(c) each goes back to its group and notifies all the agents there of their final collection point.

Lemma 3.12. Protocol GR-Even guarantees that $(k-2)$ agents will either rendezvous in the same node or gather within distance 1 .

Proof. If only one collection point was formed, the protocol GR-Even is correct for the same reasons as protocol GR-ODD.

If two collection points were formed then two collectors moving in opposite directions will eventually meet, decide on the final collection point (or edge) and inform about that all other followers.

This completes the proof of Theorem 3.5. The time and movement costs of Protocol GREVEn can be easily determined:

Theorem 3.13. Protocol GR-Even terminates in time at most $5(n-2)$. 
Proof. If only one collection point was formed, the reasoning is the same as in the proof of Theorem 3.9, and the time complexity is at most $5(n-2)$.

Assume now that two collection points were formed at a distance $d$ from each other (traversing the part of the ring not containing $\mathrm{BH}$ ). Let $t_{1}$ be the time when the last collection point was formed and let $s$ be the number of edges explored at that moment. Let $t_{2}$ be the time when the two collectors met, and $t_{3}$ be the time when all agents gathered at the common collection point or edge. Clearly, $t_{1} \leq 3 s$, and $t_{2} \leq t_{1}+d / 2+2(n-2-s)$ (since the collectors that meet travel towards each other, and at most $n-2-s$ edges must be traversed using cautious walk). It takes less than $d$ time steps for the collectors to come back to their collection points to inform the followers (they could have met quite asymmetrically), and $d / 2$ steps for everybody to come to the new common collection point (recall, it is in the middle between the two old collection points); hence, $t_{3} \leq t_{2}+3 / 2 d$. Combining together we obtain $t_{3} \leq 3 s+d / 2+2(n-2-s)+3 / 2 d=2(n-2)+2 d+s$. Since $d$ and $s$ are at most $n-2$, then $t_{3} \leq 5(n-2)$.

Theorem 3.14. Protocol GR-Even uses at most $3 n k / 2-k^{2} / 4+o(n k)$ moves.

Proof. If there is single collection point, the proof of Theorem 3.10 applies directly. If there are two collection points, applying Lemma 3.4 for the two groups creating collection points yields $2 \times\left(n k / 2+k^{2} / 8\right)$ moves. Again, only $O(n)$ moves will be spent by collectors. Incidentally, the cost of moving the agents from the two collection points to the final collection point (or pair of points) is $(n-2)(k-2) / 2$ regardless of the location of the final collection point, as both collection points contain the same amount of followers.

\subsection{RendezVous when $k$ is unknown}

An immediate consequence of the fact that $k$ is unknown is that, by Theorem 2.5, the ring size $n$ must be known for any non-trivial rendez-vous to be possible.

Another consequence is that, by Theorem 2.2, if we want to rendez-vous we must locate the black hole! Let us examine under what conditions and how the problem can be solved.

\subsubsection{Oriented Rings}

Theorem 3.15. Let $k \geq 4$. Then $R V(k-2)$ can always be solved.

To prove this theorem we design a protocol, called SHADOw, quite different from the ones used when $k$ is known.

We associate with each contiguous block of explored nodes a group of agents expanding that block until either (1) the explored block contains $n-1$ nodes (in which case a final collection phase is initiated, collecting the agents into a designated collection point) or (2) the block merges with a neighbouring explored block (in which case the corresponding groups of agents are combined into one group expanding the new, bigger block).

The group of agents expanding a block consists of at least one and at most four agents. The agents associated with a group are of two kinds: explorers and shadows - at most one of each type for each direction. The task of the explorers is to expand the explored block in the opposite directions. The shadows scan the explored block between the explorers, counting the size of the block. Their goal is to detect when the block contains $n-1$ explored nodes. Each node remembers which types of agents have visited it so far. 
At the beginning, each explored block consists of a single node containing an agent starting as a $R$-explorer. As the blocks grow, they eventually touch and their agents are combined, maintaining the following rules:

- A two-agent block (i.e. created by merging two one-agent blocks) has one $R$-explorer and one L-explorer.

- A three-agent block has two explorers (one in each direction) and a $R$-shadow.

- A four-agent block has two explorers and two shadows (one in each direction).

- Any additional agents (i.e. if merging two four-agent blocks) become passive.

The main technical difficulty arises from the fact that the whole process is distributed and the agents are not immediately aware when their block collides with another block. In addition, both ends of a block might collide with neighbouring blocks in about the same time, complicating the coordination between the agents of the block.

The technique used can be seen as aging of agents from initial $R$-explorer through $L$-explorer, $R$-shadow, L-shadow and finally passive. The aim is to have only one agent of each type per explored block - whenever an agent learns that there is a more extreme (to the right for Ragents, to the left for L- agents) agent of the same type, the agent is aged. The actual algorithm has few exceptions to this rule, as sometimes the aging has to be delayed in order to inform waiting shadows to recompute the size of the explored block.

Protocol Shadow:

Agent states: $R$-explorer, L-explorer, $R^{*}$-shadow, $R$-shadow, $L^{*}$-shadow, L-shadow, passive, collector. collector and shadow agents maintain counter size.

\section{Algorithm for agent $a$ :}

- $\boldsymbol{R}$-explorer: Move right using cautious walk as needed until a node $v$ already visited by a different $R$-explorer is entered. Become $L$-explorer.

- L-explorer: Move left using cautious walk as needed until a node $v$ already visited by an $L$-explorer is entered. Become $R^{*}$-shadow and set size $e_{a}$ to 0 .

- $\boldsymbol{R}^{*}$-shadow: Move right until the last safe node $v$ is reached, decrementing size ${ }_{a}$. If $v$ has already been visited by a $R$-shadow, set size $e_{a}$ to 0 and become $L^{*}$-shadow. If the $R$-shadow is waiting at $v$, wake him up.

If $v$ has not been visited by a $R$-shadow, become $R$-shadow.

- $\boldsymbol{R}$-shadow: (Starting at the rightmost safe node in its explored part.) If size $_{a}=0$ (i.e. you left this node, travelled across the explored block and returned, and the $R$-explorer meanwhile did not explore a new node), wait until waken up by a $R^{*}$-shadow or the $R$-explorer (which just explored the node to the right and returned to make the link safe).

In the latter case, become $R^{*}$-shadow, otherwise set size $e_{a}=0$ and move left until the last safe node is reached, counting in size $e_{a}$ the number of nodes traversed.

If size $_{a}=n-1$ then become collector else become $R^{*}$-shadow. 

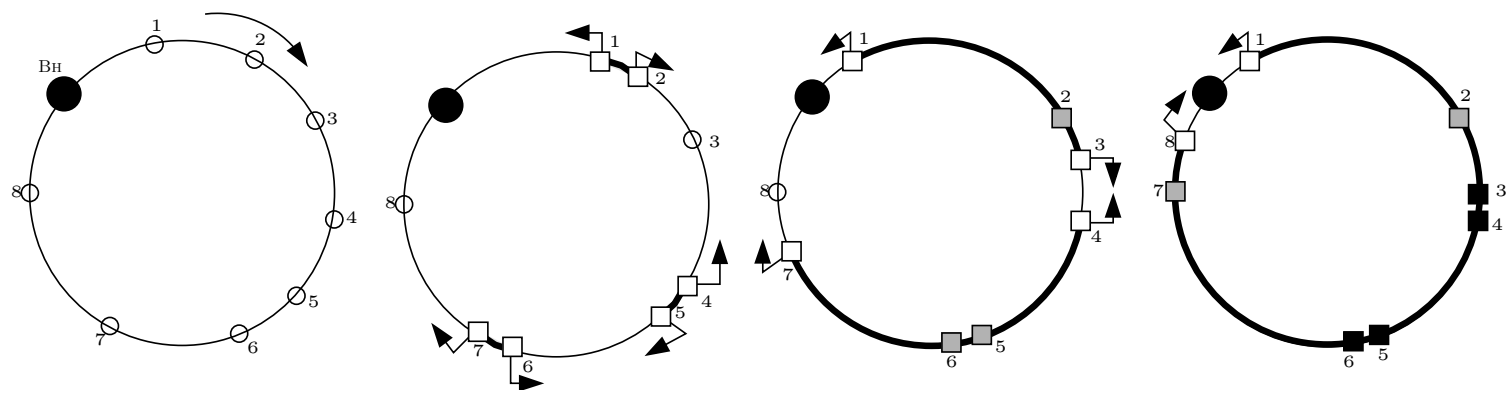

Figure 3: Protocol Shadow, where the ring is assumed to be oriented clockwise. The empty circles represent active agents; the white squares are the explorers, the grey squares the shadows, and the black squares the passive agents. The fat line evidences the segments delimited by the explorers. The numbers are placed only to clarify how the agents move, and are not used at all during the computation.

- $\boldsymbol{L}^{*}$-shadow: Move left until the last safe node $v$ is reached, decrementing size . If $v$ has already been visited by a $L$-shadow, become passive. If the $L$-shadow is waiting at $v$, wake him up.

If $v$ has not been visited by a $L$-shadow, become $L$-shadow.

- L-shadow: Analogous to $R$-shadow, but with reversed directions.

- collector: A collector agent traverses the explored part and collects all the agents on the way (if an agent meets a collector, it stops its activity and follows the collector). Once the whole explored part has been traversed (collector counts $n-1$ links), $k-2$ agents have been collected and have gathered at the same place. There is a technical detail: it can happen that both shadows can become collectors. In that case, the gathering point is the node where they meet (or, if they crossed each other on a link, the right endpoint of that link).

- passive: Wait to be collected by a collector.

Lemma 3.16. Within finite time all nodes will be explored, there will be only one R-explorer and one L-explorer, and they will both enter the black hole.

Proof. Consider the rightmost agent. From construction, it will remain a $R$-explorer until it reaches the BH. Any other $R$-explorer will eventually become $L$-explorer. The leftmost of those will remain $L$-explorer and will eventually enter the $\mathrm{BH}$, all others will become $R^{*}$-shadow. Of those, the rightmost one will eventually become $R$-shadow, any remaining one will become $L^{*}$ shadow. Finally, out of those, the leftmost one will become $L$-shadow, all others will become passive.

Let $v$ be an arbitrary node. If there was an agent to the left of $v$ in the initial configuration, the closest such agent $a$ will reach $v$ as $R$-explorer. If there were no agents to the left of $v$, the leftmost $L$-explorer would eventually explore it.

Lemma 3.17. Eventually, at least one agent will become collector. 
Proof. Consider the rightmost $R$-shadow a (since $k \geq 4$ and from the proof of Lemma 3.16 we know that there will be a $R$-shadow). From construction, a will remain $R$-shadow ${ }^{1}$ until it becomes a collector or itself is collected by a collector. It remains to be proven that $a$ will not be blocked forever waiting for the next node to the right to be explored.

Applying Lemma 3.16 we know that eventually there will be a single explored block. Consider the last step leading to an explored block of $n-1$ nodes: If the explored block reaches $n-1$ nodes by exploring the rightmost node, either $a$ is waiting in the rightmost safe node (in which case it will be awaken by the $R$-explorer and will traverse the $n-1$ explored nodes to become collector) or is currently scanning the explored block and will eventually return to the (new) rightmost explored node, notice that the block has been expanded and re-scan it. A similar argument applies to the leftmost $L$-shadow if the explored block reaches $n-1$ nodes by exploring the leftmost node. Finally, if the explored block reaches size $n-1$ by merging of two blocks, either $a$ will detect that directly, or will be awaken by the $R^{*}$-shadow created by the collision of the blocks and re-scan to become collector.

The proof of Theorem 3.15 now follows quite straightforwardly from the construction of collector agents.

Let us now examine the time costs of this protocol.

Theorem 3.18. The protocol SHADOw terminates in at most 8(n-2) time steps.

Proof. Let $s_{0}$ be the position of the leftmost agent (measured from the BH, going right) and $s_{1}$ be the position of the second leftmost agent. Then at time $3\left(s_{1}-s_{0}\right)$ the leftmost agent becomes $L$-explorer and enters the $\mathrm{BH}$ at time $3 s+4\left(s_{1}-s_{0}\right)$. If only the leftmost agent woke up spontaneously, it takes $3\left(n-s_{0}-1\right)$ time step for the right exploration to reach the $\mathrm{BH}$. Therefore, all nodes are explored by the time step $\max \left(3 s+4\left(s_{1}-s_{0}\right), 3\left(n-s_{0}-1\right)<4(n-2)\right.$. Note also that by time step $3\left(n-s_{0}-1\right)$ there is a single $R$-explorer, as all other $R$-explorer agents would have entered a node already visited by a $R$-explorer. This also means that by time $3\left(n-s_{0}-1\right)+n-2<4(n-2)$, there will remain only one $L$-explorer, i.e. all other agents became shadows. This means by time $5(n-2)$ there will remain a single right shadow $\left(R^{*}\right.$-shadow or $R$-shadow) and by time $6(n-2)$ there will be a single left shadow.

If a shadow initiates scan after time $4(n-2)$, it will detect that $n-1$ nodes have been explored and will become collector. The algorithm would then terminate in additional at most $n-2$ steps.

An unsuccessful scan (without becoming collector) takes at most $2(n-3)$ steps, therefore a successful scan would be initiated at time less then $6(n-2)$ and the algorithm would terminate by time $8(n-2)$.

Let us stress that protocol SHADOW solves the black hole location problem (by Lemma 3.17). This means that we have obtained a significant improvement over the $O(n \log n)$ time complexity of the existing protocols for the black hole search.

Theorem 3.19. The protocol SHADOw locates the $\mathrm{BH}$ using at most $4 n^{2}+n k-k^{2} / 2+O(n)+$ $o\left(k^{2}\right)$ moves.

Proof. First, note that at most $O(n)$ moves are performed by the explorers.

Let us now count the number of moves executed by all agents in states $R^{*}$-shadow and $R$-shadow. Let us call recharge the events of a $R$-shadow being awaken by a $R^{*}$-shadow or $R$ explorer. Note that there are at most $2 n$ recharge events for all right shadows, as in each event

\footnotetext{
${ }^{1}$ more precisely, alternating between $R$-shadow and $R^{*}$-shadow
} 
either a $R^{*}$-shadow becomes a $L^{*}$-shadow, or the $R$-explorer has made progress. Moreover, a $R$-shadow (or $R^{*}$-shadow) can make at most $2(n-2)$ moves between recharges - if it is not recharged, it turns into $L^{*}$-shadow. This means that the total number of moves by all agents in states $R$-shadow and $R^{*}$-shadow can be bound by $2 n^{2}$.

Using analogous arguments, it is easy to see that the total number of moves by all agents in states $L$-shadow and $L^{*}$-shadow is at most $2 n^{2}$ as well.

The total cost of collection can be bound by $n k-k^{2} / 2+o\left(k^{2}\right)$ using similar arguments as in the proof of Theorem 3.10.

Summing together yields the theorem.

\subsubsection{Unoriented Rings}

Interestingly, we discover for the unoriented case better conditions than those we have found when $k$ was known instead of $n$.

\section{Theorem 3.20.}

1. If $k$ odd or $n$ even, $R V(k-2)$ can always be solved

2. If $k$ even and $n$ is odd, $R V(p)$ can not be solved for $p>\lfloor(k-2) / 2\rfloor$; however, $R V(\lfloor(k-2) / 2\rfloor)$ can always be solved.

3. $G(k-2,1)$ can always be solved.

We prove Theorem 3.20 constructively by presenting a protocol Un-Shadow. The main ideas of protocol SHADOw carry over to protocol UN-SHADOw, however, several technicalities have to be dealt with differently due to lack of agreement on orientation. Again, we colour the agents red a blue, according to their initial orientation. In general, the agents follow protocol SHADOW ignoring the agents of the different colour, however these exceptions apply:

1. when a $R$-explorer comes to a node already visited by a $L$-explorer of the opposite colour, it becomes $R^{*}$-shadow.

2. when a $L$-explorer comes to a node already visited by a $R$-explorer of the opposite colour, it becomes $R^{*}$-shadow.

3. the L- and R- for shadows are used only for aging purposes. The tests are made against the shadows working in the same direction. For example, when a red $R^{*}$-shadow a arrives to the last explored node $v$ in its direction, it checks whether $v$ has been visited by a red $R$-shadow or blue $L$-shadow. If yes, a becomes a $L^{*}$-shadow (and possibly awakes the shadow at $v$, regardless of its colour), otherwise $a$ becomes $R$-shadow.

4. when (if) two collectors cross each other on an edge, they may not agree on which endpoint to meet (if the situation is symmetric), therefore only $G(k-2,1)$ is guaranteed, not $R V(k-2)$.

Note that if all agents are of the same colour, the protocol UN-SHADOW behaves exactly as protocol SHADOw and its correctness follows. Therefore, in the rest we assume there is at least one agent of each colour. Let us also assume that red $R$-explorer moves to the right and blue $R$-explorer moves to the left.

Lemma 3.21. Within finite time, all nodes will be explored. 
Proof. Consider an initial configuration. Let $v$ be an arbitrary node and let $a_{l}$ and $b_{l}$ be the closest and second closest agents to $v$ from the left, and $a_{r}$ and $b_{r}$ be the closest agents from the right, respectively. (Not all of them might be present, but at least one side has at least two agents - because $k \geq 4$.)

From construction, if $a_{l}$ or $b_{l}$ is a red agent, it will travel to the right and either reach $v$ or meet a left-moving agent that already visited $v$. Similarly, $v$ will be explored if $a_{r}$ or $b_{r}$ is blue.

W.l.o.g. assume the left side has at least two agents and both $a_{l}$ and $b_{l}$ are blue. $b_{l}$ starts moving left as a $R$-explorer and when it enters the starting node of $a_{l}$, it will become $L$-explorer and start moving right. Therefore, $v$ will be eventually explored either by $b_{l}$ or by an agent coming from the right.

Lemma 3.22. Within finite time, an agent will become collector.

Proof. Since the only time an agent is waiting is either when it is passive, or as an $R$-shadow or $L$-shadow, and because $k \geq 4$, eventually there will be a shadow for the right direction (ref $R$-shadow or blue $L$-shadow) and a shadow for the left direction. The rightmost such right shadow and the leftmost left shadow will not age any more, because other shadows will age due to their rightmost/leftmost mark.

When all nodes become explored (Lemma 3.21 tells us that this will eventually happen), at least one of those extreme agents will be awaken, traverse the explored nodes and become collector (using the same arguments as in Lemma 3.17).

Consider first the case when $k$ is odd or $n$ is even.

Lemma 3.23. There will be a unique collection point.

Proof. If there is only one collector, there will be a single collection point - when the collector travelled $n-1$ nodes. If there are two collectors, when they meet, they can agree on the collection point (i.e. directly opposite the $\mathrm{BH}$ ).

Corollary 3.24. $k-2$ agents will gather in the collection point.

Consider now the case when $k$ is even and $n$ is odd.

Lemma 3.25. If $k$ is even and $n$ is odd then $R V(p)$ can not be solved for $p>(k-2) / 2$; however, $R V((k-2) / 2)$ and $G(k-2,1)$ can be achieved.

Proof. Consider a ring where $k / 2$ red entities are positioned at one side of the black hole, and $k / 2$ blue agents are on the other side. Consider now a synchronous scheduler that sends the two agents closest to the black hole to move towards the black hole; under this scheduler there will always be the same number of agents at the two sides of the ring.

From Lemma 3.22 we know that there will be a collector, however there might be two of them - one for each direction. A single collector would collect $k-2$ agents. If there are two collectors, at the moment they meet (or cross each other on a link), both $R V((k-2) / 2)$ and $G(k-2,1)$ is achieved.

Combining Lemmas 3.24 and 3.25 yields Theorem 3.20

Using the same approach as in the proof of Theorem 3.18 we get

Theorem 3.26. The protocol UN-SHADOw for unoriented rings terminates in at most $8(n-2)$ time steps. 
Since the UN-SHADOW differs from the protocol SHADOW only by accelerated aging and final collection, the proof of Theorem 3.19 carries almost without changes also for UN-SHADOW.

Theorem 3.27. The protocol Un-Shadow locates the $\mathrm{BH}$ using at most $4 n^{2}+n k-k^{2} / 2+$ $O(n)+o\left(k^{2}\right)$ moves.

\section{Conclusions}

We need some real conclusions, so far we have just this short discussion how to extend the results. We have assumed that the agents are initially dispersed, with each agent in a different homebase.

If at the beginning there are two or more agents at the same node, we immediately group them and elect a "leader" among them (e.g. using the mutual exclusion in writing to the whiteboard); the others in the group will "follow the leader" (except on unsafe links).

With careful attention to details, all the proposed protocols can be modified so to handle this situation.

\section{References}

[1] S. Alpern. The Rendezvous Search Problem. SIAM Journal of Control and Optimization, Volume 33, 673 - 683, 1995.

[2] S. Alpern and V. Baston and S. Essegaier. Rendezvous Search on a Graph. Journal of Applied Probability, 36, No.1, 223-231, 1999.

[3] E.J. Anderson and R.R.Weber. The Rendezvous Problem on Discrete Locations. Journal of Applied Probability, 28, 839-851, 1990.

[4] E. Arkin, M. Bender, S. Fekete, and J. Mitchell. The freeze-tag problem: how to wake up a swarm of robots. In 13th ACM-SIAM Symposium on Discrete Algorithms (SODA '02), pages 568-577, 2002.

[5] L. Barrière, P. Flocchini, P. Fraigniaud, and N. Santoro. Capture of an intruder by mobile agents. In 14th ACM Symp. on Parallel Algorithms and Architectures (SPAA '02), 200-209, 2002 .

[6] L. Barrière, P. Flocchini, P. Fraigniaud, and N. Santoro. Election and rendezvous in fully anonymous systems with sense of direction. In 10th Colloquium on Structural Information and Communication complexity (SIROCCO '03), 17-32, 2003.

[7] A. Dessmark, P. Fraigniaud and A. Pelc. Deterministic rendezvous in graphs. In 11th Annual European Symposium on Algorithms (ESA'03) 2003.

[8] S. Dobrev, P. Flocchini, G. Prencipe, and N. Santoro. Mobile agents searching for a black hole in an anonymous ring. In 15th Int. Symposium on Distributed Computing (DISC 2001), 166-179, 2001.

[9] S. Dobrev, P. Flocchini, G. Prencipe, and N. Santoro. Searching for a black hole in arbitrary networks. In 21st ACM Symposium on Principles of Distributed Computing (PODC '02), 153-162, 2002. 
[10] S. Dobrev, P. Flocchini, R. Král'ovič, G. Prencipe, P. Ružička, and N. Santoro. Searching for a black hole in hypercubes and related networks. In 6th International Conference on Principles of Distributed Systems (OPODIS '02),171-182, 2002.

[11] P.Flocchini, E. Kranakis, D. Krizanc, N. Santoro, and C. Sawchuk. Multiple Mobile Agent Rendezvous in a Ring. In LATIN '04 (accepted for publication).

[12] F. Hohl. A Framework to Protect Mobile Agents by Using Reference States. In International Conference on Distributed Computing Systems (ICDCS '00), 2000.

[13] E. Kranakis, D. Krizanc, N. Santoro, and C. Sawchuk. Mobile Agent Rendezvous in a Ring. In 23rd International Conference on Distributed Computing Systems (ICDCS'03), 2003.

[14] W.S. Lim and A. Beck and S. Alpern. Rendezvous search on the Line with More Than Two Players. Operations Research, 45, pp. 357-364, 1997.

[15] P. Panaite and A. Pelc. Exploring unknown undirected graphs. Journal of Algorithms, 33:281-295, 1999.

[16] T. Sander and C. F. Tschudin. Protecting mobile agents against malicious hosts. In: Mobile Agents and Security, LNCS 1419, pp. 44-60, 1998.

[17] L.C. Thomas amd P.B. Hulme. Searching for Targets Who Want to be Found. Journal of the Operations Research Society, 48, Issue 1, pp. 44-50, 1997.

[18] Jan Vitek and Giuseppe Castagna. Mobile Computations and Hostile Hosts. In: D. Tsichritzis (Ed.), Mobile Objects, University of Geneva, pp. 241-261, 1999.

[19] X. Yu and M. Yung. Agent Rendezvous: A Dynamic Symmetry-Breaking Problem. In ICALP '96, LNCS 1099, 610-621, 1996. 